\title{
DETECTION OF FOCAL EPILEPTIFORM ACTIVITY IN THE EEG: AN SVD AND DIPOLE MODEL APPROACH
}

\author{
Bart Vanrumste ${ }^{1,2, *}$, Richard D. Jones ${ }^{1,2,3}$ and Philip J. Bones ${ }^{1}$ \\ ${ }^{1}$ Department of Electrical and Computer Engineering, University of Canterbury, Christchurch, New Zealand \\ ${ }^{2}$ Department of Medical Physics \& Bioengineering, Christchurch Hospital, Christchurch, New Zealand \\ ${ }^{3}$ Department of Medicine, Christchurch School of Medicine and Health Science, Christchurch, New Zealand \\ *E-mail: bart.vanrumste@elec.canterbury.ac.nz, Fax. +64-3-364 2761
}

\begin{abstract}
An algorithm has been developed for detection of epileptiform activity in the EEG. The EEG is divided into overlapping epochs, which undergo two steps. The first is singular value decomposition (SVD) which identifies the number of uncorrelated active sources in an epoch. In the second step, EEG dipole source analysis, using a single dipole model, is applied to the EEG. This yields dipole parameters and a relative residual energy (RRE). The detection algorithm triggers an EEG epoch when SVD indicates a dominant source and the RRE is low. The algorithm is applied to simulated EEG generated by two sources which are synchronously and asynchronously active. For the synchronous case the critical measure is the RRE whereas for the asynchronous case both the SVD and RRE are critical. The algorithm has also been applied to real EEG containing two spikes and an eye-blink artifact. The SVD indicated a dominant active source and the RRE was low for all three events. These preliminary results demonstrate the potential of the method for detection of spikes and seizures with a focal origin.

Keywords- spike/seizure detection, singular value decomposition, dipole localization.
\end{abstract}

\section{INTRODUCTION}

Patients with epilepsy often undergo long-term EEG monitoring (2-4 days) resulting in large amounts of digital EEG. Manual detection of epileptic activity is impractical and, hence, automatic detection algorithms are employed. The first algorithms applied ad hoc criteria to imitate the human observer and are called 'mimetic approaches' [1]. These algorithms investigate the temporal aspect of the EEG at each channel separately. It has been shown that incorporating spatial context can substantially reduce the number of false detections $[2,3]$. Different criteria need to be applied when dealing with interictal and ictal waves.

The location of an epileptogenic focus can be estimated through EEG source analysis. This technique finds the dipole parameters which generate scalp electrode potentials which best fit the measured potentials. The algorithm presented in this paper detects epochs of EEG which estimate a dipole as a reasonable underlying source. The algorithm was introduced in [4] where it is shown that for one $20 \mathrm{~min}$ EEG a sensitivity of $80 \%$ and a specificity of $60 \%$ could be obtained. The algorithm utilizes only the spatial aspects of the EEG and can be used for seizure and spike detection. In addition to detection, the dipole parameters can provide valuable information on the location of the epileptogenic zone.

\section{METHODS}

The EEG epoch $\mathbf{V} \in \mathbb{R}^{\mathrm{m} \times \mathrm{n}}$ is decomposed by SVD into $\mathbf{U} \cdot \mathbf{s} \cdot \mathbf{W}^{\mathbf{T}}$ with $m$ 'potential distributions' found in the columns of $\mathbf{U} \in \mathbb{R}^{\mathrm{m} \times \mathrm{m}}, m$ corresponding time courses in the columns of $\mathbf{W} \in \mathbb{R}^{\mathbf{n} \times m}$, and the singular values $s_{i}$ found on the diagonal entries of diagonal matrix $\mathbf{s} \in \mathbb{R}^{\mathrm{m} \times \mathrm{m}}$. The $s_{i}$ values are ordered such that the one with the largest value has the smallest index $i$. An assumption made is that most of the signal energy of an epileptic event is contained in the first component of the SVD. The measure

$$
S=\frac{s_{1}^{2}}{\sum_{i=1}^{m} s_{i}^{2}}
$$

represents this fraction. The measure $S$ is used to determine times when a single SVD-component is dominant in the EEG.

A second assumption is that the underlying sources generating an epileptic events can be modelled by a dipole. The relative residual energy,

$$
R R E=\frac{\left\|\mathrm{U}_{* 1}-\mathbf{V}_{\text {model }}\right\|^{2}}{\left\|\mathbf{U}_{* 1}\right\|^{2}},
$$

with $\mathbf{V}_{\text {model }}$ being the potentials generated by a dipole in a three-shell spherical model, is used to validate this assumption. The RRE gives the fraction of energy which cannot be explained by the dipole.

Fig. 1 presents $10 \mathrm{~s}$ of simulated EEG generated by two dipole sources, one located in the occipital and one in temporal brain region (only 2 channels shown). From 1-5 s both sources were synchronously active at $5 \mathrm{~Hz}$. From 6-10 s the temporal source is active at $5 \mathrm{~Hz}$ and the occipital source at $9 \mathrm{~Hz}$. For both segments the amplitude ratio between the temporal and occipital source increases from 1 to 3 . Fig. 2 shows a 10-s segment of EEG containing two spikes, and an eye-blink artifact. Both EEGs are recorded at 19 electrodes placed according to the international $10-20$ system. The EEG is divided into $500-\mathrm{ms}$ epochs for simulated EEG and 250-ms epochs for real EEG respectively, 

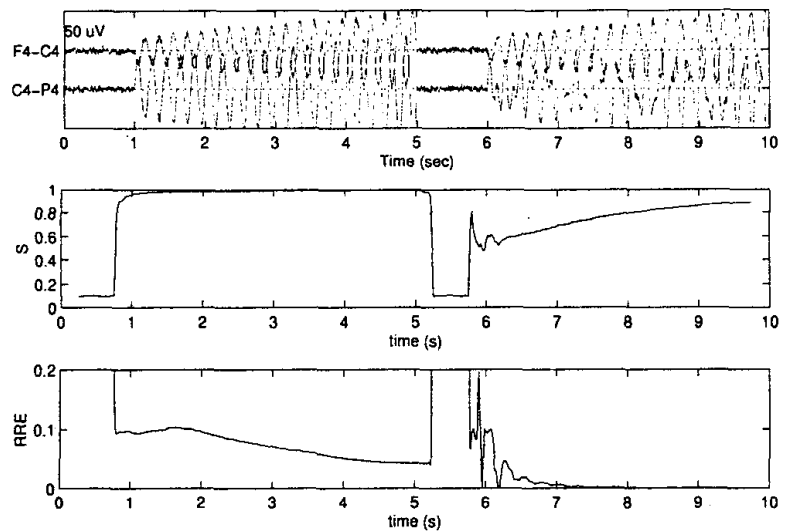

Figure 1. A 10-5 segment of simulated EEG and corresponding $S$ and RRE.
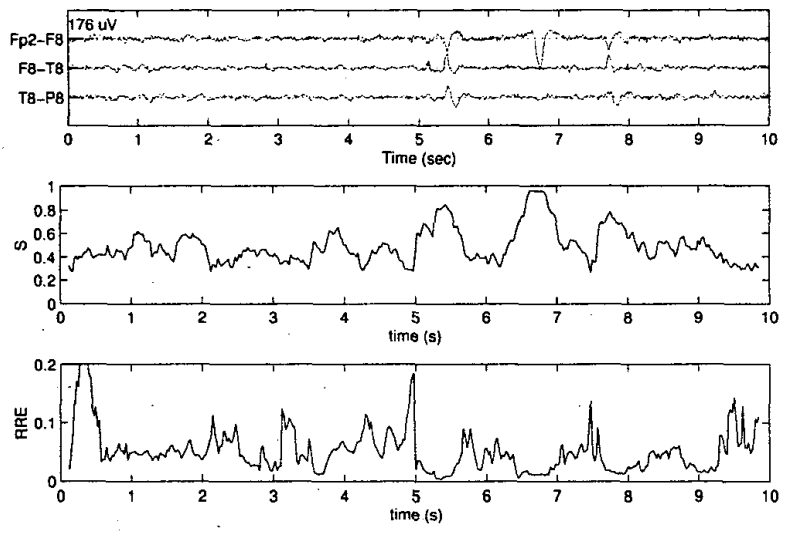

Figure 2. A 10-s segment of EEG and corresponding $S$ and RRE. The EEG contains focal spikes at $5.3 \mathrm{~s}$ and $7.8 \mathrm{~s}$ and an eye-blink at $6.8 \mathrm{~s}$.

with each epoch shifted 50 -ms relative to, and hence substantially overlapping, the previous epoch. For each epoch $S$ and RRE were calculated.

\section{RESULTS}

In Fig.1, $S$ is high for the synchronous segment while RRE decreases as the temporal source becomes more dominant. For synchronous activity, RRE is the critical measure.

For the asynchronous activity, $S$ increases and RRE decreases as the temporal source becomes dominant. RRE becomes effectively zero at the point where $\mathrm{U}_{* 1}$ equals the potential distribution associated with the dipole source in the temporal region.

For both spikes and the eye-blink, $S$ has a high value compared to the remaining EEG (Fig. 2) indicating a strongly dominant singular value. The RRE has a low value for all three events, suggesting dipoles as good models of the underlying sources. However, it is notable that the RRE value is also low at other time instances.

\section{DISCUSSION}

Our current research is aimed at finding optimal thresholds for $S$ and RRE in terms of sensitivity and specificity for epileptiform activity.

Not surprisingly, focal artifacts such as eye-blinks are also detected with the current algorithm. However it is considered that these can be removed by inspecting the locations of dipoles associated with them.

For now, the source localization is performed in an iterative way which prevents the algorithm from working in real-time. However, it has been shown that artificial neural networks can replace the iterative procedure making real-time applications possible [5].

Detected epochs come with corresponding dipole parameters. Thus if the detected events are questionable, due, say, to coming from a deep focus, it will be possible to enhance the EEG from the suspected source by way of a 3D spatial filter [6] focussed on the event's dipole parameters. The enhanced questionable events can then be reexamined and more definitively assigned as being epileptiform or artifact.

Preliminary determination of performance of a detection algorithm based on SVD and dipole localization is promising but validation on a larger EEG datasets is required.

\section{REFERENCES}

[1] J. Gotman and P. Gloor, "Automatic recognition and quantification of interictal epileptic activity in the human scalp EEG," Electroenceph. Clin. Neurophysiol., vol. 41, pp. 513-529, 1976.

[2] A. Dingle, R. Jones, G. Carroll, and R. Fright, "A multistage system to detect epileptiform activity in the EEG," IEEE Trans. Biomed. Eng., vol. 40, pp. 1260-1268, 1993.

[3] C. James, R. Jones, P. Bones, and G. Carroll, "Detection of epileptiform discharges in EEG by a hybrid system comprising mimetic, self-organized artificial neural networks, and fuzzy logic stages," Clin. Neurophysiol. vol. 110, pp. 2049-2063, 1999.

[4] G. Van Hoey, B. Vanrumste, R. Van de Walle, P. Boon, I. Lemahieu, M. D'Havé, and K. Vonck, "Detection and localization of epileptic brain activity using an artificial neural network for dipole source analysis," in Proc. EUSIPCO2000 Conf., Tampere, Finland, 2000.

[5] G. Van Hoey, J. De Clercq, B. Vanrumste, R. Van de Walle, M. D'Havé, I. Lemahieu, and P. Boon, "EEG dipole source localization using artificial neural networks," Phys. Med. Biol., vol. 45, pp. 997-1011, 2000.

[6] D.-M. Ward, R. Jones, P. Bones, and G. Carroll, "Enhancement of deep epileptiform activity in the EEG via 3D adaptive spatial filtering," IEEE Trans. Biomed. Eng., vol. 46, pp. 707-716, 1999. 